\title{
Learning the "Special Note": Evidence for a Critical Period for Absolute Pitch Acquisition
}

\author{
Frank A. Russo
}

Queen's University

Deborah L. Windell

Queen's University

\section{Lola L. Cuddy}

Queen's University

Please Cite:

Russo, F. A., Windell, D. L., \& Cuddy, L. L. (2003). Learning the "special note": Evidence for a critical period for absolute pitch acquisition. Music Perception: An Interdisciplinary Journal, 21(1), 119-127.

doi:10.1525/mp.2003.21.1.119

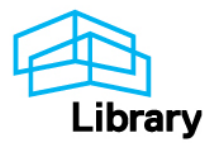




\title{
Learning the "Special Note": Evidence for a Critical Period for Absolute Pitch Acquisition
}

\author{
FRANK A. RUSSO, DEBORAH L. WINDELL, \\ \& LOLA L. CUDDY \\ Queen's University
}

\begin{abstract}
Children (3-6 years old) and adults were trained for 6 weeks to identify a single tone, $\mathrm{C}_{5}$. Test sessions, held at the end of each week, had participants identify $\mathrm{C}_{5}$ within a set of seven alternative tones. By the third week of training, identification accuracy of children 5-6 years old surpassed the accuracies of children 3-4 years old and adults. Combined with an analysis of perceptual strategies, the data provide strong support for a critical period for absolute pitch acquisition.
\end{abstract}

Received July 12, 2003, accepted August 1, 2003

A focal question in the theoretical debate concerning experiential and early training during a critical period of neurodevelopment is necessary and possibly sufficient (see, e.g., Baharloo, Johnston, Service, Gitschier, \& Freimer, 1998; Brown, Sachs, Cammuso, \& Folstein, 2002; Chin, 2003; Levitin \& Zatorre, 2003; Takeuchi \& Hulse, 1993; Vitouch, 2003, and references cited in these articles). Through isolating a critical period for AP, and understanding the accompanying neural and cognitive development, the interaction of genetic and environmental influences may be discovered (Zatorre, 2003).

To date, the main evidence for a critical period for AP is correlational. AP possessors, unlike nonpossessors, typically report early, preschool, music training. Direct empirical evidence for a critical period is scant. Some adult nonpossessors have shown improvements in tone-naming with training (e.g., Brady; 1970; Cuddy, 1968, 1970; Levitin, 1999), a finding suggesting a form of latent AP, but evidence is lacking whether adults can achieve the "true" AP of early acquirers (Levitin \& Zatorre, 2003). Direct support for

Address correspondence to Frank A. Russo, Department of Psychology, University of Toronto, Mississauga, Ontario, L5L 1C6, Canada. (e-mail: frusso@utm.utoronto.ca)

ISSN: 0730-7829. Send requests for permission to reprint to Rights and Permissions, University of California Press, 2000 Center St., Ste. 303, Berkeley, CA 94704-1223. 
the critical period hypothesis requires evidence of superior AP acquisition by children compared with adults under identical training and testing methods. Only a few experimental studies have tackled this comparison, and the results of these studies are equivocal.

Cohen and Baird (1990) trained preschool children and adults to discriminate a major triad (e.g., C E G) from nontriads presented over five octaves. Identification tests, in which participants then had to distinguish the major triad from another triad on an absolute basis (e.g., C E G versus C F A) were too difficult for both age groups, and no clear age-related differences emerged. Crozier (1997) trained preschool children and adolescents at single-tone $\left(A_{4}\right)$ acquisition. After training, children showed greater improvement than adolescents at reproduction (humming $\mathrm{A}_{4}$ ). Children tended to show greater improvement than adolescents on a perception task (picking $\mathrm{A}_{4}$ from a set of three alternatives), but the difference was not significant.

The present study offers clear support for children's enhanced ability to learn to identify a single tone on an absolute basis. It follows the study by Crozier (1997) but altered several methodological procedures to increase reliability and the likelihood of obtaining age-related differences should they exist. First, whereas Crozier's study compared children's performance with adolescents, the present study compared children's performance with adults. Second, whereas Crozier's training method consisted of all members of a group humming $\mathrm{A}_{4}$ together, the present training consisted of individual practice on tone identification. Third, whereas Crozier's testing procedure presented $\mathrm{A}_{4}$ on each trial in the context of two other tones that differed from trial to trial, the present test contained the same set of seven alternatives on each trial. One of the seven was the tone to be identified. As Crozier acknowledged, with his procedure the correct response $\mathrm{A}_{4}$ could be determined by interrelating successive trials. The present modification, as well as preventing a between-trial cue to the correct response, yielded similar performance levels for children and adults at the start of training. Crozier's adolescent sample started training at a significantly higher performance level than did the children, an unexplained finding that introduced further complexities to interpretation of his data.

\section{Method}

PARTICIPANTS

Participants were volunteers from the Queen's University community-eight children (six girls and two boys) and eight adults (four women and four men). Age range for children was 3-6 years $(M=4.6$ years) and for adults was $21-39$ years $(M=29.5$ years $)$. Training was conducted by a parent for each child and a partner for each adult. The educational background of the children's parents ranged from college to a doctoral degree with a 
median level of a bachelor's degree. The educational background of the adults ranged from high-school graduate to a bachelor's degree with a median level of 3 years of university. No participant had formal music training.

\section{MATERIALS AND PROCEDURES}

Each participant and each parent/partner was issued one hand-held flag, and each parent/partner was issued one Master Key pitch pipe, model AZL number 6012. The pipe produced complex tones with substantial energy at odd harmonics up to about the 25th harmonic and less than 1\% variation in fundamental frequency (as measured by Soundswell Signal Workstation, 1997).

Four components-overview, pretest, training, and test-were conducted in participants' homes. The overview and pretest occurred on the first day, followed by training and testing for 6 weeks.

For the overview, the participant was told that there was a "special note" (i.e., $\mathrm{C}_{5}$ ) to be learned. This "special note" was played and the participant was asked to hum the tone (or octave equivalent) along with the pitch pipe. The experimenter and the participant (or the participant's parent) then reviewed the training procedure and time commitment. The overview took approximately 15 minutes.

The overview was followed by the pretest, conducted by one of the authors (D.L.W.). On each trial, participants were asked to discriminate the "special note" from six alternatives. Seven tones- $\mathrm{G}_{4}, \mathrm{~A}_{4}, \mathrm{~B}_{4}, \mathrm{C}_{5}, \mathrm{D}_{5}, \mathrm{E}_{5}$, and $\mathrm{F}_{5}$ - were played in random order. Duration of each tone was approximately $1.5 \mathrm{~s}$, with intertone duration approximately $2 \mathrm{~s}$. Participants were asked to raise a flag whenever they thought they heard the "special note" and to guess if not sure. If the participant failed to respond to one of the tones on a trial, or responded to several tones, the trial was repeated. No feedback was given. Ten trials were conducted, with the same seven tones in different random order on each trial.

Training sessions were held 1 to 4 times per week $(M=3.13, S D=0.51)$ during a 6 -week period, and a practice log, recording details of the training session, was kept by the parent/ partner. Each training session involved the presentation of a set of four tones from the $\mathrm{C}$ major scale within the range $\mathrm{G}_{4}$ to $\mathrm{F}_{5}$. In Weeks 1 and 2, the tone-set consisted of two $\mathrm{Cs}$ and two other randomly selected tones. In Weeks 3-6, the tone-set consisted of one $\mathrm{C}$ and three other randomly selected tones.

Each trial comprised three phases in which the parent/partner played the tone-set on the pitch pipe. In Phase 1, the parent/partner raised a flag whenever the "special note" was played. In Phase 2, both the parent/partner and participant raised flags for the "special note". In Phase 3, only the participant raised a flag for the "special note." Each session contained 10 trials, with the order of tone presentation independently randomized for each trial.

Testing sessions were conducted by D.L.W. and were identical to the pretest, except that feedback was available after the testing session was complete. Testing always occurred after training for the week was complete and never on the same day as training. Each testing session lasted approximately 20 minutes.

\section{Results and Discussion}

An alpha level of .05 was used for all statistical tests. The percentage of trials in which $\mathrm{C}_{5}$ was correctly identified as the "special note" was scored for each participant for each of seven testing sessions. Mean scores on the pretest did not differ significantly between children $(M=16.25, S E=1.83)$ and adults $(M=15, S E=2.67), t(14)=1.38, n s$, and the combined score did not differ significantly from chance, $t(15)=0.85, n s$. 
Scores for all eight children and seven of the eight adults improved from pretest to final test. Scores for the younger children ( 3 and 4 years old-3 girls and 1 boy) on the final test ranged from $30 \%$ to $60 \%$ correct, whereas scores for the older children ( 5 and 6 years old -3 girls and 1 boy) ranged from $80 \%$ to $100 \%$ correct. Scores for the adults on the final test ranged from $10 \%$ to $100 \%$. The mean percentage correct for each test session for the younger children, older children, and adults is shown in Figure 1. After the third week of training, the older children, on average, outperformed both the younger children and the adults. Practice logs of the older children included remarks such as "really seems to be getting it" and "having fun guessing the C."

A mixed analysis of variance (ANOVA) was conducted with age group as the between-subjects variable and test session as the within-subjects variable. Test session was significant, $F(6,78)=22.02, p<.0001$, as well as the interaction between test session and age group, $F(12,78)=2.49, p<.01$.

Response distributions across test sessions (the percentage of trials on which each tone was selected as the "special note") are shown in Figure 2. For both younger and older children, the course of learning the "special note" proceeded from equal preference for each of the test tones to a gradual

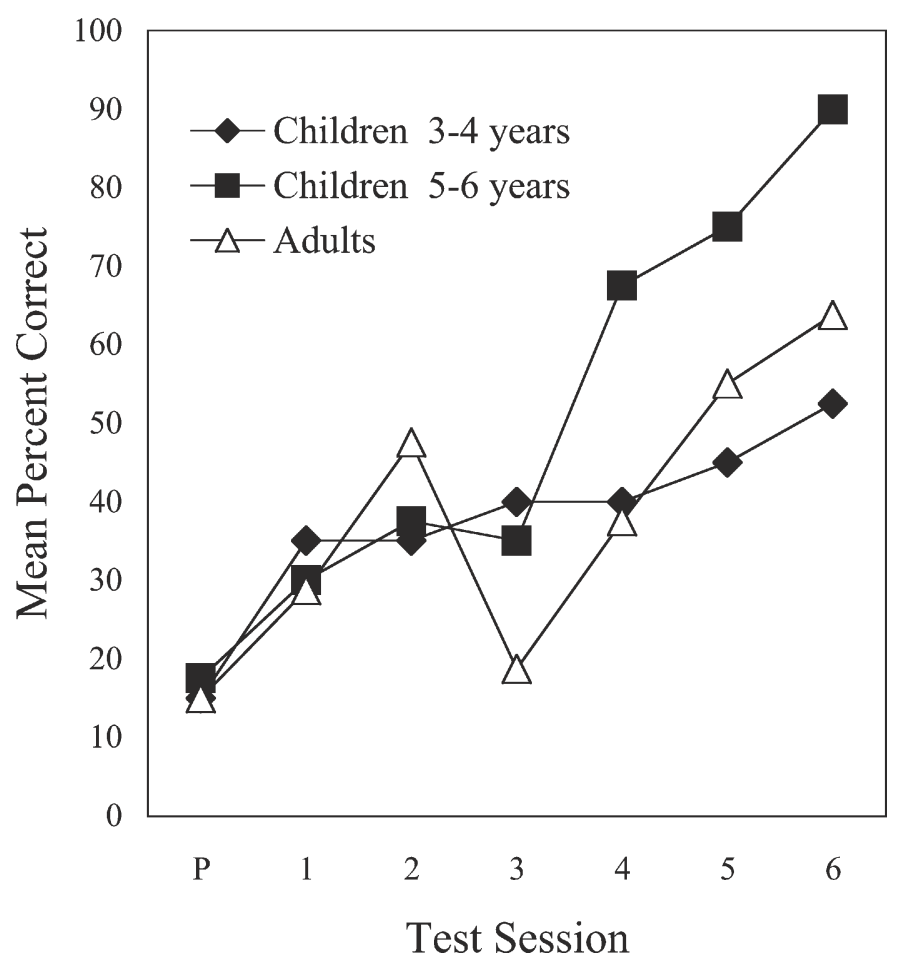

Fig. 1. Mean percent correct across test sessions for children (3-4 years old, 5-6 years old) and adults. 
targeting of $\mathrm{C}_{5}$. In contrast, for the adults the course of learning began with an initial preference for a subset of the seven alternatives, followed by a preference for different members of the subset on different test sessions.
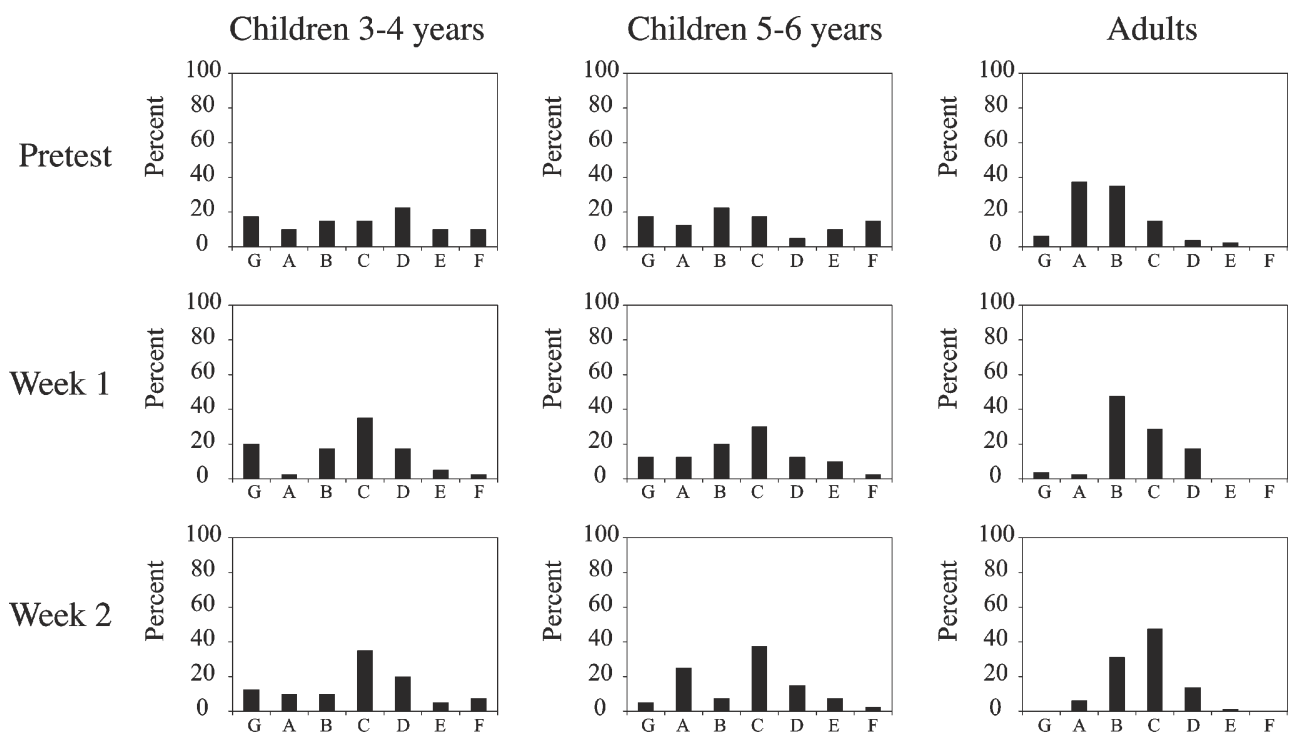

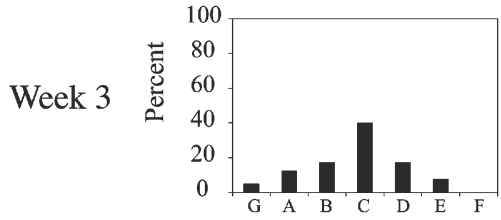
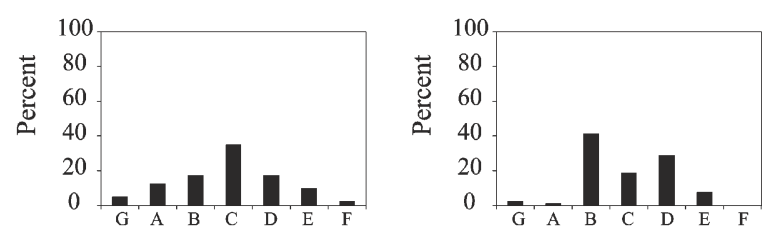

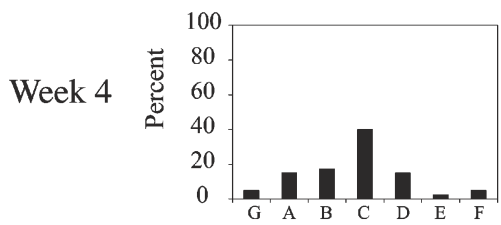
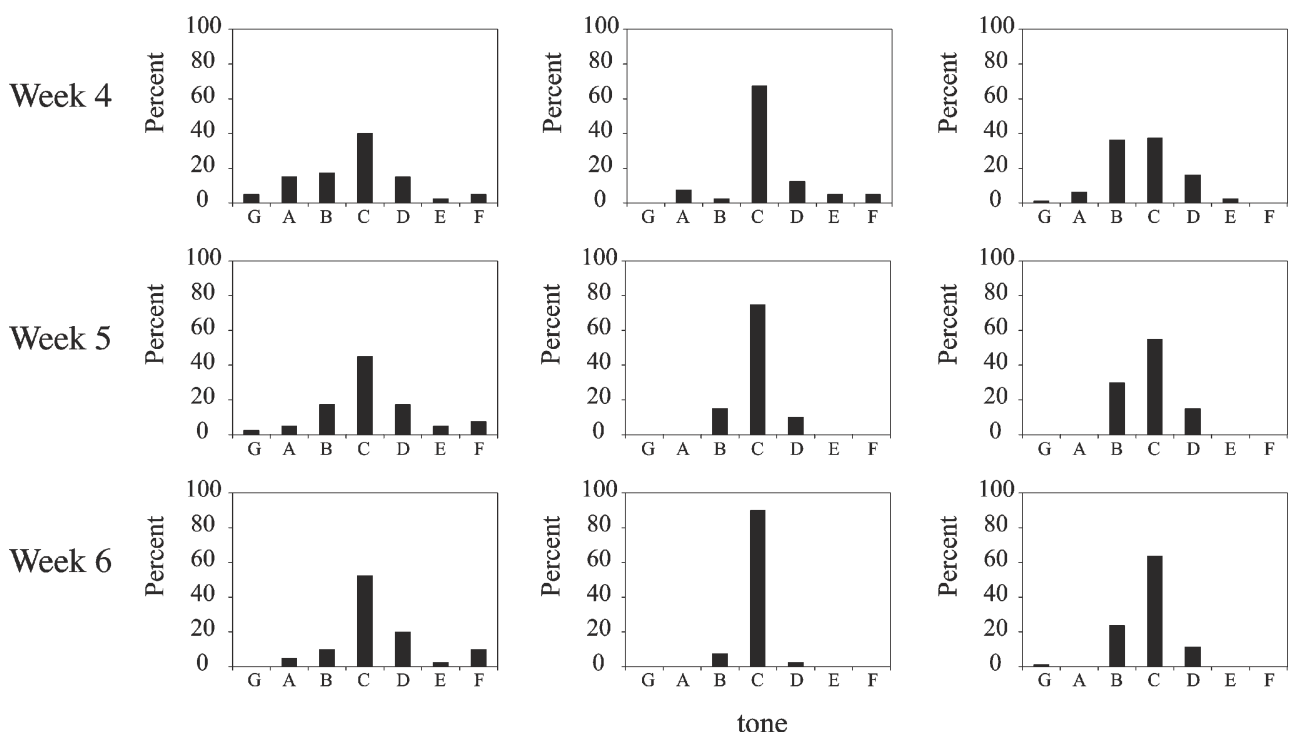

Fig. 2. Percentage of trials on which each tone was selected as the "special note" across test sessions for children (3-4 years old, 5-6 years old) and adults. 
Although $\mathrm{C}_{5}$ within this subset was preferred on Weeks 2, 5, and 6, the incorrect tone $\mathrm{B}_{4}$ was preferred on Week 3 , thus leading to a decrease in overall accuracy (see Figure 1).

Response distributions shown in Figure 2 were typical for individual participants. Chi-square tests compared the obtained response distributions for each participant for each session against four theoretical distributions: (1) random-equal preference for each of the seven tones; (2) pitch-region-equal preference for a three-tone subset of tones with no clear preference for any one member of the subset; (3) incorrect mode-preference for one tone that was not the "special note"; and (4) correct mode-preference for the "special note." Theoretical distributions were tested in order, (1) to (4); the distribution for which the chi-square value was not significant, $\chi^{2}(6) \leq 12.59$, was considered the best fitting.

For all children, pretest data were best fit by the random distribution. Six children attained correct mode by the end of training. Three (two older and one younger) moved from a random distribution to correct mode. Three (two older and one younger) moved from random through pitch region to correct mode. Incorrect mode never appeared in the children's data. Five adults attained correct mode by the final testing session. However, the progression for the adults was more variable and typically involved an alternation between pitch region and incorrect mode. Pitch region was about three times more likely to occur for adults than for children, and all adults had at least one session categorized as incorrect mode.

Individual data for children and adults, including the percent correct and the best-fitting response type for each testing session, are given in Appendixes $\mathrm{A}$ and $\mathrm{B}$, respectively.

In summary, after 3 weeks of training, children 5-6 years old were on average remarkably more adept at distinguishing a single tone from a set of alternative tones than were children 3-4 years old or adults. This early age corresponds to that reported by Baharloo et al. (1998) and others for the typical age at which AP possessors report first music training. The older children, and some of the younger children, adopted a perceptual strategy of moving the pitch boundaries for identification from the full range of tones to a narrow category around the single tone. In contrast, adults focused on a subset of tones within the set of alternatives. They adopted a short-term strategy - to select one member of the subset during testing and to persevere with that choice. If the choice was wrong, the strategy led to poor performance. Although adults revealed a stable absolute pitch memory within a session, they had more difficulty than children retaining the fixed boundaries of a single tone across training sessions.

Future directions should include testing of a wider range of children's ages, testing delayed recall, and training and testing identification of 
additional tones. Individual differences deserve further study. Within age groups, the consistent training method did not produce identical performance in all participants, a result hinting at a genetic component. Nevertheless, the overall difference between children and adults in perceptual strategy is striking. To our knowledge, the results provide the first unequivocal experimental support for a critical period in which tone-label acquisition is privileged. ${ }^{1}$

\section{References}

Baharloo, S., Johnston, P. A., Service, S. K., Gitschier, J., \& Freimer, N. B. (1998). Absolute pitch: An approach for identification of genetic and nongenetic components. American Journal of Human Genetics, 62, 224-231.

Brady, P. T. (1970). Fixed-scale mechanism of absolute pitch. Journal of the Acoustical Society of America, 48, 883-887.

Brown, W., Sachs, H., Cammuso, K. \& Folstein, S. E. (2002). Early music training and absolute pitch. Music Perception, 19, 595-597.

Chin, C. S. (2003). The development of absolute pitch: A theory concerning the roles of music training at an early developmental age and individual cognitive style. Psychology of Music, 31, 155-171.

Cohen, A. J., \& Baird, K. (1990). Acquisition of absolute pitch: The question of critical periods. Psychomusicology, 9, 31-37.

Crozier, J. B. (1997). Absolute Pitch: Practice makes perfect, the earlier the better. Psychology of Music, 25, 110-119.

Cuddy, L. L. (1968). Practice effects in the absolute judgment of pitch. Journal of the Acoustical Society of America, 43, 1069-1076.

Cuddy, L. L. (1970). Training the absolute identification of pitch. Perception \& Psychophysics, 8, 265-269.

Levitin, D. J. (1999). Memory for musical attributes. In P. R. Cook (Ed.), Music, cognition, and computerized sound: An introduction to psychoacoustics (pp. 209-227). Cambridge, MA: MIT Press.

Levitin, D. J., \& Zatorre, R. J. (2003). On the nature of early music training and absolute pitch: A reply to Brown, Sachs, Cammuso, and Folstein. Music Perception, 21, 105110.

Soundswell Signal Workstation [computer software]. (1997). Sweden: AB Nyvalla DSP. http://www.nyvalla.dsp.se.

Takeuchi, A. H., \& Hulse, S. H. (1993). Absolute pitch. Psychological Bulletin, 113, 345361.

Vitouch, O. (2003). Absolutist models of absolute pitch are absolutely misleading. Music Perception, 21, 111-118.

Zatorre, R. J. (2003). Absolute pitch: A model for understanding the influence of genes and development on neural and cognitive function. Nature Neuroscience, 6, 692-695.

1. This research was supported by a discovery grant to L. L. Cuddy from the Natural Sciences and Engineering Council of Canada. We thank the families and individual participants for their cooperation and enthusiasm. We also thank the anonymous reviewers of this report. All authors contributed equally to this project. 


\section{Appendix A}
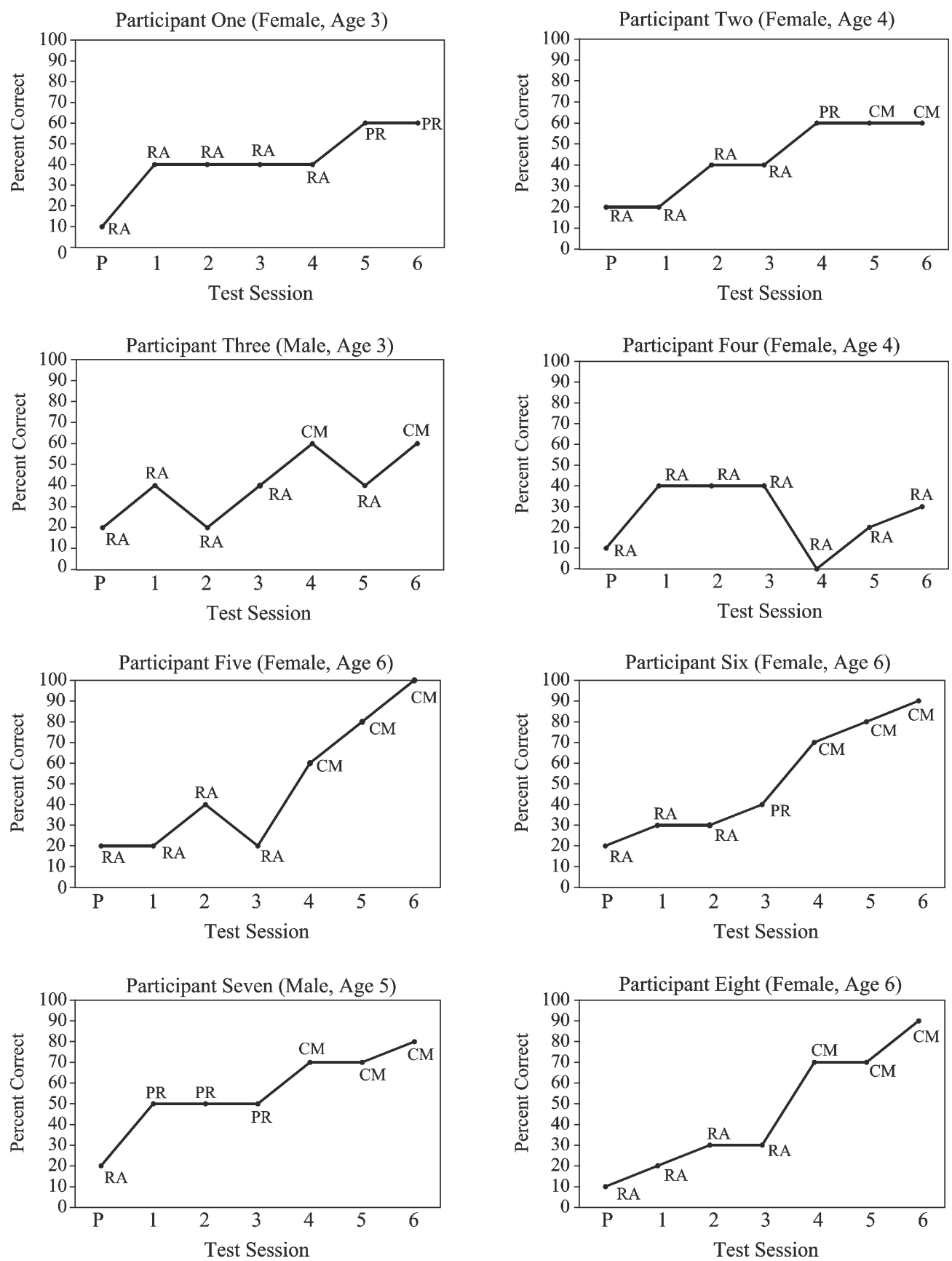

Figure A. Percent correct and best-fitting response distribution $(\mathrm{RA}=$ random, $\mathrm{PR}=$ pitch region, $\mathrm{IM}=$ incorrect mode, $\mathrm{CM}=$ correct mode) across test sessions for individual children. 


\section{Appendix B}
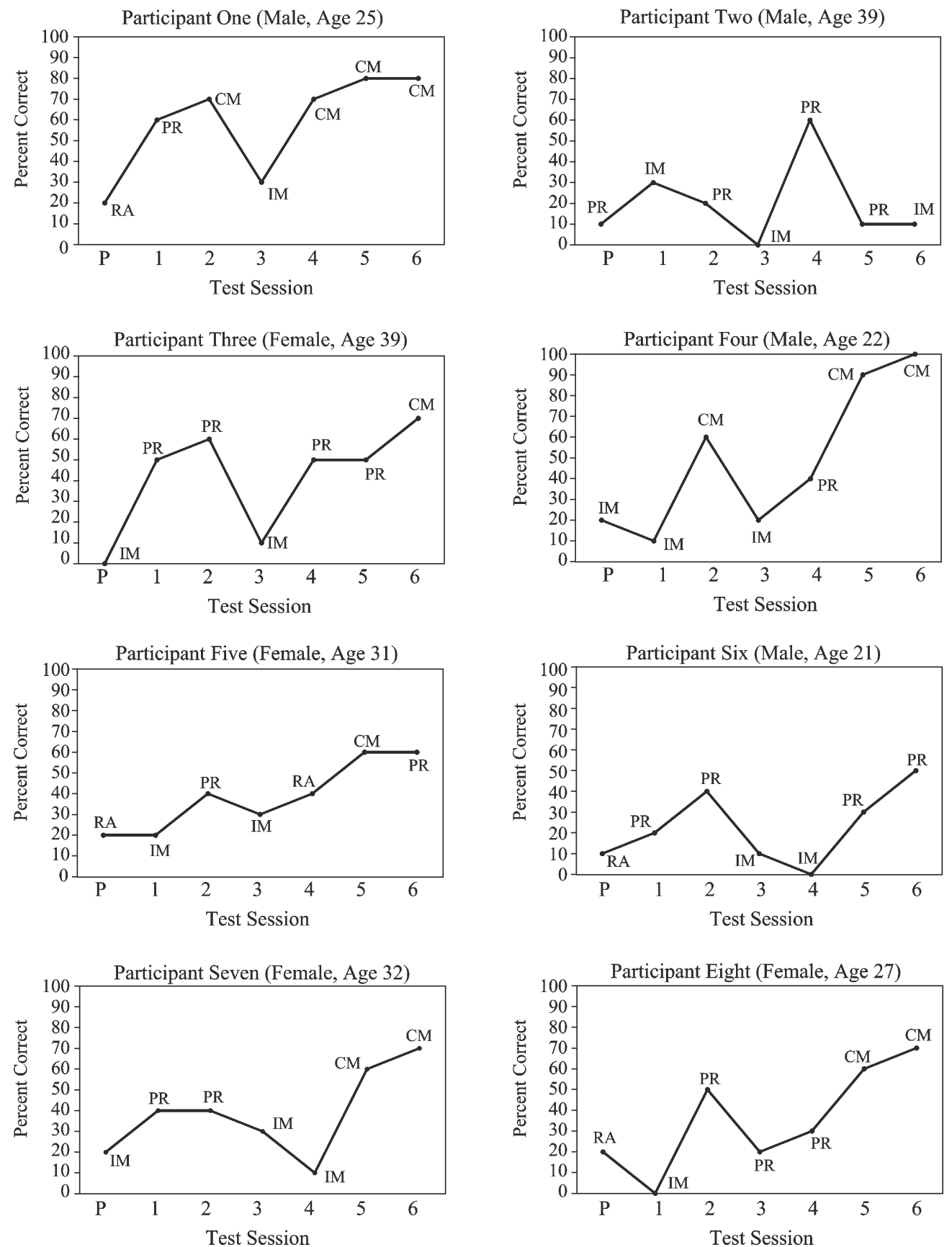

Figure B. Percent correct and best-fitting response distribution (RA = random, $\mathrm{PR}=$ pitch region, $\mathrm{IM}=$ incorrect mode, $\mathrm{CM}=$ correct mode) across test sessions for individual adults. 